\title{
СТРУКТУРА АДАПТИВНОЙ СИСТЕМЫ КОМПЕНСАЦИИ РЕАКТИВНОЙ МОЩНОСТИ
}

\section{A. В. Ярошевич 1}

\author{
${ }^{1}$ К. т. н., доцент, доцент кафредры автоматизации технологических процессов и производств учреждения образования
} «Брестский государственный технический университет», Брест, Беларусь, e-mail: ya-v@tut.by

\begin{abstract}
Реферат
Статья посвящена построению моделей адаптивной системы компенсации реактивной мощности.

Для решения задачи синтеза системы выбрана схема компенсатора реактивной мощности с аналоговым вычислением ёмкости конденсаторов.

В статье отражены следующие вопросы:

- проведён анализ схем компенсаторов для снижения потерь от реактивной мощности в электрических сетях;

- разработана графическая информационная модель компенсатора реактивной мощности с аналоговым вычислением ёмкости конденсаторов как системы автоматического управления;

- произведён выбор элементов системы;

- создана оптимальная структурная схема с оптимизацией параметров.
\end{abstract}

Ключевые слова: математическая модель, система автоматического управления, компенсация реактивной мощности.

\section{THE STRUCTURE OF THE ADAPTIVE POWER COMPENSATION SYSTEM}

\section{Abstract}

\section{A. V. Yaroshevich}

The article is devoted to the construction of models of an adaptive reactive power compensation system.

To solve the problem of synthesizing the system, a reactive power compensator circuit with analog calculation of the capacitance of the capacitors was chosen. The article reflects the following questions:

- the analysis of compensator circuits to reduce losses from reactive power in electrical networks was carried out;

- developed a graphical information model of the reactive power compensator with analog calculation of the capacitance of capacitors as an automatic control system;

- made a selection of system elements;

- an optimal structural diagram with optimization of parameters was created.

Keywords: mathematical model, automatic control system, reactive power compensation.

\section{Введение}

Ранее было принято считать, что из-за относительно коротких фидеров городских низковольтных распределительных сетей, небольшой присоединенной мощности и рассредоточения нагрузок проблемы компенсации индуктивной мощности на городских сетях не существует.

Если принять во внимание, что за последнее десятилетие расход электроэнергии на $1 \mathrm{~m}^{2}$ жилищного сектора увеличился втрое, средняя статистическая мощность силовых трансформаторов городских муниципальных сетей достигла 325 кBA, а зона использования трансформаторной мощности сместилась в сторону увеличения и находится в пределах 250...400 кВА , то это утверждение вызывает сомнение [1].

Обработка графиков нагрузки, снятых на вводе многоквартирного жилого дома, показала: в течение суток средние значения коэффициента мощности $(\cos \varphi)$ менялось от 0,88 до 0,97 , а фазные - от 0,84 до 0,99. Таким образом, учитывая высокую плотность коммунально-бытовой нагрузки, постоянное наличие в перетоках мощности индуктивной составляющей приводит к значительным потерям электроэнергии в распределительных сетях крупных городов.

\section{Структура графической модели компенсатора реактивной} мощности

Сложность решения данного вопроса во многом связана с неравномерным потреблением реактивной мощности по отдельным фазам, затрудняющая применение традиционных для промышленных сетей установок покрытия индуктивной мощности на базе трехфазных батарей конденсаторов, управляемых регулятором, установленным в одной из фаз компенсируемой сети.

В таблице представлен перечень электрооборудования среднестатистической семьи из трех человек.

\begin{tabular}{|c|c|c|c|c|}
\hline $\begin{array}{l}\text { Погребители } \\
\text { энергии }\end{array}$ & $\underset{\mathbf{\text { KBT }}}{\text { Мощность, }}$ & $\begin{array}{l}\text { Кол- } \\
\text { во, шт }\end{array}$ & $\begin{array}{c}\text { Среднесуточное } \\
\text { время работы, ч/сут }\end{array}$ & $\begin{array}{c}\text { Месячный } \\
\text { расход } \\
\text { эл.энергии, кВт·ч }\end{array}$ \\
\hline Холодильник & 1 & 1 & 2 (с уетом пауз) & 60 \\
\hline Телевизор & 0,08 & 1 & 5 & 12 \\
\hline $\begin{array}{l}\text { Стиральная } \\
\text { машина }\end{array}$ & 1,5 & 1 & 0,57 (4 ч. в нед.) & 26 \\
\hline Электрочайник & 2 & 1 & 0,25 & 15 \\
\hline $\begin{array}{l}\text { Персональный } \\
\text { компьютер }\end{array}$ & 0,15 & 1 & 2 & 9 \\
\hline Пылесос & 0,8 & 1 & 0,14 (1 ч. в нед.) & 3 \\
\hline Утюг & 1 & 1 & 0,29 (2 ч. в нед.) & 9 \\
\hline $\begin{array}{l}\text { Микроволновая } \\
\text { печь }\end{array}$ & 1 & 1 & 0,20 & 6 \\
\hline $\begin{array}{l}\text { Освещение (лампы } \\
\text { накаливания) }\end{array}$ & 0,06 & 10 & 3 & 54 \\
\hline итого & \multicolumn{4}{|l|}{194} \\
\hline
\end{tabular}

Большинство бытовых потребителей переменного тока являются потребителями и индуктивной мощности. Потребителями являются приемники электроэнергии, которые по принципу своего действия используют переменное магнитное поле: асинхронные двигатели, индукционные печи, сварочные транссрорматоры, выпрямители и т. п., а также звенья электрической сети - трансформаторы, линии электропередачи, реакторы и другое оборудование. Около 60 \% всей индуктивной мощности, связанной с образованием переменных магнитных полей, потребляют асинхронные двигатели и около $25 \%$ - транссрорматоры.

Потребление активной и индуктивной мощности всегда сопровождается потерями. В масштабе электрической системы потерями считаются мощности, расходуемые в элементах и электрооборудовании электрической сети (в воздушных и кабельных линиях, силовых трансформаторах, реакторах и в другом оборудовании понижающих 
Вестник Брестского государственного технического университета. 2021

подстанций). Заметим существенную разницу в соотношении потребления и потерь активной и индуктивной мощности. Основная часть активной мощности потребляется нагрузками и лишь незначительная (около $10 \%$ ) теряется в элементах сети. Индуктивная мощность в элементах сети и электрооборудовании обычно соизмерима по величине с активной мощностью, потребляемой нагрузками.

Передача индуктивной мощности от генераторов электростанций по электрической сети к потребителям вызывает в сети затраты активной мощности в виде потерь. Поэтому, как правило, увеличение выдачи индуктивной мощности генераторами станций с целью доставки ее потребителям нецелесообразно, а наибольший экономический эффрект достигается при размещении компенсирующих устройств вблизи потребляющих индуктивную мощность нагрузок.

Наглядное представление о сущности компенсации реактивной мощности даёт векторная диаграмма (рисунок 1) [2]. На рисунке 1, а изображена схема электрической цепи. Пусть до компенсации потребитель имел активную мощность $\mathrm{P}$, соответственно ток $I_{a}$ (отрезок $O B$ на рисунке 1 , б и реактивную мощность от индуктивной нагрузки $Q$ L с соответствующим током I L (отрезок BA). Полной мощности $S_{1}$ соответствует вектор I н (отрезок $\left.O A\right)$. Коэфффициент мощности до компенсации $\cos \varphi_{1}$

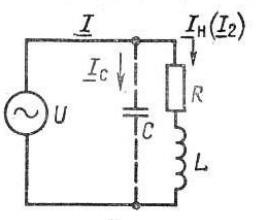

a)

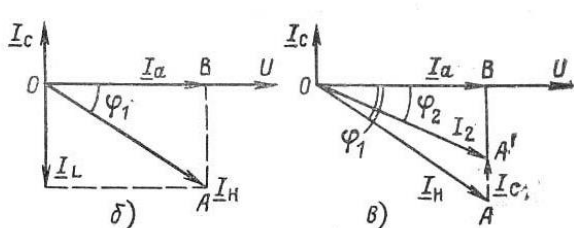

Рисунок 1 - Векторная диаграмма компенсации реактивной мощности

Векторная диаграмма компенсации представлена на рисунке 1, в. После компенсации, т. е. после подключения параллельно нагрузке конденсаторной установки с мощностью $Q \kappa($ ток $I C$ ), суммарная реактивная мощность потребителя будет уже $Q_{1}-Q_{K}\left(\right.$ ток $\left.I_{L}-I_{C}\right)$ и соответственно снизится угол сдвига фаз с $\varphi_{1}$ до $\varphi_{2}$ и повысится коэффициент мощности с $\cos \varphi_{1}$ до $\cos \varphi_{2}$. Полная потребляемая мощность при той же потребляемой активной мощности $\mathrm{P}$ (токе $\left.l_{a}\right)$ снизится с $S_{1}\left(\right.$ ток $I_{H}$ ) до $S_{2}$ (ток $I_{2}$ ) (отрезок $O A$ ). Следовательно, в результате компенсации можно при том же сечении проводов повысить пропускную способность сети при активной мощности.

Анализ многообразия методов управления компенсацией реактивной мощности (РМ) позволяет сделать следующие выводы:

1. Все методы базируются на косвенной оценке величины реактивной мощности и не обеспечивают требуемое значение $\cos \varphi$ сети.

2. Оценка компенсируемой мощности требует изучения специфических особенностей нагрузки и технологии производственных процессов.

3. Схемы управления компенсацией требуют индивидуального подхода в проектировании и наладке под конкретную ситуацию.

4. Схемы управляющих устройств компенсаторов не отличаются простотой и надёжностью.

На основе этих выводов можно сделать заключение о том, что исключить названные недостатки можно при использовании автоматического управления компенсацией реактивной мощности на основе измерения величины реактивной мощности в нагрузке или электрических параметров нагрузки, по которым можно вычислить величину компенсируемой реактивной мощности.

Промышленные устройства ступенчатого регулирования реактивной мощности построены с применением микропроцессорного контроллера и являются сложными и дорогими для массового использования в квартирных и других электрических сетях до 0,4 кВ с нагрузками до $50 \mathrm{kBT}$.

Исходя из этого, можно сформулировать основные требования к вычислителю компенсатора реактивной мощности для бытовых нагрузок:
1. Для эффективной компенсации реактивной мощности ёмкость конденсаторной батареи должна регулироваться в зависимости от фактического значения реактивной составляющей нагрузки.

2. Использование простых асимптотических формул для расчёта ёмкости позволяет построить простую надёжную схему управления ключами с аналоговым вычислителем для коммутации конденсаторов.

3. Компьютерное моделирование фазового детектора позволило выбрать схему и определить параметры сигнала для управления коммутацией компенсирующих конденсаторов.

4. Использование простой и недорогой схемы в цепях бытовой нагрузки позволит получить существенную экономию за счёт сокращения потерь при передаче электроэнергии.

Для обеспечения экономической целесообразности применения решающим параметром должна быть стоимость устройства. Стоимость определяется построением электрических схем из простых и надёжных элементов, количество которых должно быть невелико. Такой подход позволит обеспечить другое важнейшее требование небольшие габариты, позволяющие встраивать компенсаторы реактивной мощности в квартирные щиты энергоснабжения.

Способ решения этой задачи и некоторые требования к устройствам предложены в [3] путём применения аналоговой схемы вычислителя для компенсатора реактивной мощности.

При реализации схемы компенсации РМ необходимо решить следующие проблемы:

- измерение величины реактивных потерь в реальном времени;

- определение ёмкости компенсирующего конденсатора;

- коммутация к сети ёмкости из конденсаторной батареи.

Общая схема предлагаемого устройства для компенсации РМ бытового потребителя электрической энергии представлена на рисунке 2.

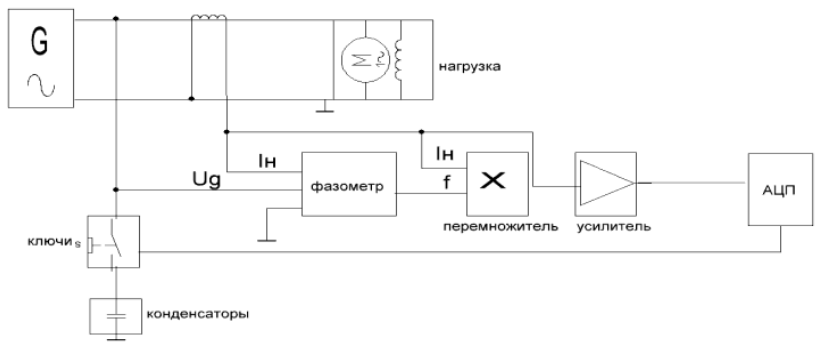

Рисунок 2 - Блок-схема системы автоматического управления (САУ) компенсации РМ

Схема компенсации РМ подключается между счётчиком электроэнергии и нагрузкой потребителя. Основными узлами схемы являются:

- схема измерения разности фаз сетевого напряжения и потребляемого тока, отражающая долю РМ в нагрузке;

- схема управления симисторными ключами для коммутации компенсирующей ёмкости. Схема включает перемножитель параметров тока нагрузки и разности фаз для реализации фрормулы определения ёмкости конденсаторов для компенсации РМ и усилитель для масштабирования полученного произведения;

- линейка симисторных ключей между конденсаторами батареи и фазным проводом;

- компенсаторная батарея конденсаторов.

В качестве измерителя тока может использоваться измерительный трансформатор или резисторный шунт.

Схему управления ключами предлагается реализовать на основе асимптотических формул для расчёта ёмкости компенсирующего конденсатора [4].

Для обоснования предлагаемой асимптотической формулы получены следующие соотношения.

При средневзвешенном значении $\cos \varphi=0,85$ погрешность от представления $\operatorname{tg} \varphi \approx \varphi$ (рад) не превысит 0,1 от значения $\operatorname{tg} \varphi$. Тогда соотношение активной и реактивной мощности $\operatorname{tg} \varphi=Q / P$ можно представить формулой $Q / \mathrm{P} \approx \varphi$. 
Погрешность от представления $\cos \varphi \approx 1-\varphi$ не превысит 0,12 значения $\cos \varphi$, тогда активную мощность можно представить формулой $P \approx U I(1-\varphi)$, реактивную мощность $Q \approx U I(1-\varphi) \varphi$.

Индуктивная мощность нагрузки, подлежащая компенсации

$$
Q\left\llcorner\approx U I_{H}(1-\varphi) \varphi,\right.
$$

где $U$ - напряжение сети

$I_{H}$ - ток в нагрузке.

С другой стороны, ёмкостная мощность

$$
Q_{C}=I_{C}^{2} /(2 \pi f C)
$$

где $f$ - частота сети,

$C$ - ёмкость компенсирующего конденсатора,

$I_{C}=I_{H} \sin \varphi \approx I_{H} \varphi$ - ток ёмкости.

Отсюда можно получить соотношение

$$
C \approx \frac{I_{H} \varphi}{2 \pi f U(1-\varphi)}(\Phi),
$$

а с учётом зачений $f=50 Г ц, U=220 \mathrm{~B}$

$$
C \approx \frac{K I_{H} \varphi}{1-\varphi}(\Phi)
$$

где $k \approx 0,000015$.

Для вычисления значения ёмкости в мкФ коэффицциент $\kappa=15$ тогда

$$
C \approx \frac{15 I_{H} \varphi}{1-\varphi}(\text { МКФ). }
$$

Допустив, что $1-\varphi \approx 1$, получим $C \approx 15 I_{H} \varphi$.

Эта асимптотическая формула является основой для построения схемы управления ключами.

Структурная схема адаптивной САУ компенсацией реактивной мощности приведена на рисунке 3.

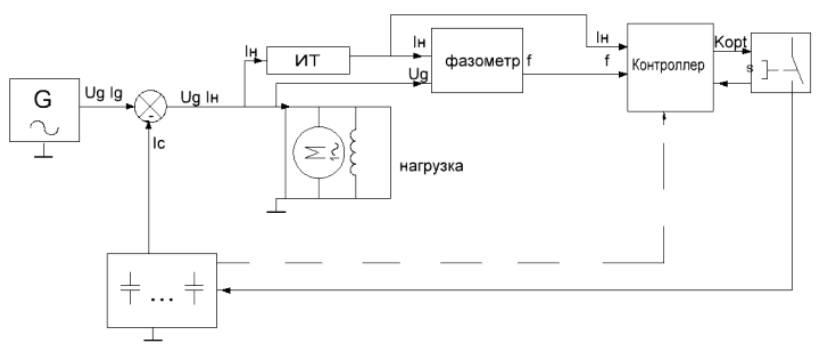

Рисунок 3 - Структурная схема САУ КРМ

Генератор G питает нагрузку системы синусоидальным напряжением 220 В 50Гц. Сигналы Ug, Ig поступают на вход элемента сравнения. В данном случае элемент сравнения выполняет функции векторной алгебры, создавая векторные суммы комплексов токов. Нагрузка меняет параметры сигнала тока на $/ H=l g-I C$, где конденсаторный ток создаётся из условия резонанса токов индуктивной нагрузки и компенсирующих конденсаторов.

Измеритель тока ИТ формирует сигнал ІН, который с сигналом $U g$ подаётся на фазометр, на выходе которого напряжение $f$, пропорциональное разности фаз питающего напряжения и тока нагрузки.
Контроллер принимает на себя функции блока умножения, усилителя и аналогово-цифрового преобразователя (АЦП). Блок умножения перемножает сигналы тока и разности фаз в соответствии с формулой для расчёта емкости конденсаторов. Сигнал $\mathrm{C}$ на выходе блока определяет компенсирующую ёмкость. Усилитель масштабирует сигнал до уровня, требуемого АЦП для формирования кода $K$ управления ключами коммутации конденсаторов компенсирующей батареи.

Коммутация в соответствии с кодом $K$ приведёт к неравномерной нагрузке на ключи и работающие конденсаторы, что существенно снизит надёжность элементов. Кроме того, при ограниченной долговечности элементов важно контролировать их состояние и учитывать его при управлении коммутацией. С этой целью в контур управления введён микропроцессорный контроллер. Функции контроллера состоят в анализе состояния симисторов коммутатора и конденсаторов, выборе алгоритма коммутации по многим критериям и инсрормирования о потере работоспособности устройства. На выходе контроллера формируется сигнал управления ключами Kopt.

Блок коммутаторов создаёт электрическую цепь для тока через блок компенсирующих конденсаторов.

\section{Заключение}

Представление компенсатора реактивной мощности адаптивной системой автоматического управления позволяет построить модель в терминах теории автоматического управления.

\section{Список цитированных источников}

1. Компания «Матик-электро» [Электронный источник]. - 2011. Режим доступа: www.matic.ru.

2. Шишкин, С. А. Реактивная мощность потребителей и сетевые потери электроэнергии // Энергосбережение № 4. - 2004.

3. Регулятор реактивной мощности с аналоговым вычислителем. Рэспубліка Беларусь / ПАТЭНТ на карысную мадэль № 8066 / Аўтар Ярошевич А. В. / Зарэгістравана ў Дзяржаўным рэестры карысных мадэляў 2011.12.15.

4. Ярошевич, А. В. Схема компенсации реактивной мощности в квартирных электрических сетях / А. В. Ярошевич // Вестник Брестского государственного технического университета. Брест: БрГТУ. 2011. - №5(71): Физика, математика, информатика. - С. 66-67.

\section{References}

1. Kompaniya «Matik-elektro» [Elektronnyj istochnik]. - 2011. - Rezhim dostupa: www.matic.ru.

2. SHishkin, S. A. Reaktivnaya moshchnost' potrebitelej i setevye poteri elektroenergii // Energosberezhenie № 4. - 2004.

3. Regulyator reaktivnoj moshchnosti $s$ analogovym vychislitelem. Respublika Belarus' / PATENT na karysnuyu madel' № 8066 / Ay̆tar Yaroshevich A. V. / Zaregistravana y̌ Dzyarzhay̌nym reestry karysnyh madelyay̆ 2011.12.15.

4. Yaroshevich, A. V. Skhema kompensacii reaktivnoj moshchnosti v kvartirnyh elektricheskih setyah / A. V. Yaroshevich // Vestnik Brestskogo gosudarstvennogo tekhnicheskogo universiteta. Brest: BrGTU. 2011. - №5(71): Fizika, matematika, informatika. - S. 66-67.

Материал поступил в редакцию 01.03.2021 